\title{
Distribution of the local density of states as a criterion for Anderson localization: Numerically exact results for various lattices in two and three dimensions
}

\author{
Gerald Schubert, ${ }^{1}$ Jens Schleede, ${ }^{2}$ Krzysztof Byczuk, ${ }^{3}$ Holger Fehske, ${ }^{2}$ and Dieter Vollhardt ${ }^{4}$ \\ ${ }^{1}$ Regionales Rechenzentrum Erlangen, Friedrich-Alexander-Universität Erlangen-Nürnberg, 91058 Erlangen, Germany \\ ${ }^{2}$ Institut für Physik, Ernst-Moritz-Arndt Universität Greifswald, 17487 Greifswald, Germany \\ ${ }^{3}$ Institute of Theoretical Physics, University of Warsaw, 00-681 Warsaw, Poland \\ ${ }^{4}$ Theoretical Physics III, Center for Electronic Correlations and Magnetism, Institute for Physics, \\ University of Augsburg, 86135 Augsburg, Germany \\ (Received 15 February 2010; revised manuscript received 16 March 2010; published 8 April 2010)
}

\begin{abstract}
Numerical approaches to Anderson localization face the problem of having to treat large localization lengths while being restricted to finite system sizes. We show that by finite-size scaling of the probability distribution of the local density of states (LDOS) this long-standing problem can be overcome. To this end we reexamine this method, propose numerical refinements, and apply it to study the dependence of the distribution of the LDOS on the dimensionality and coordination number of the lattice. Particular attention is given to the graphene lattice. We show that the system-size dependence of the LDOS distribution is indeed an unambiguous sign of Anderson localization, irrespective of the dimension and lattice structure. The numerically exact LDOS data obtained by us agree with a log-normal distribution over up to ten orders of magnitude and thereby fulfill a nontrivial symmetry relation previously derived for the nonlinear $\sigma$ model.
\end{abstract}

DOI: 10.1103/PhysRevB.81.155106

PACS number(s): 71.23.An, 72.15.Rn, 71.30.+h, 05.60.Gg

\section{INTRODUCTION}

The Anderson transition from extended to localized states in disordered systems has been a subject of intense theoretical and numerical investigations in the past, ${ }^{1-5}$ and is also of great current interest. ${ }^{6-9}$ In particular, with the discovery of graphene $^{10}$ a truly two-dimensional (2D) system is now available in which localization effects can be studied experimentally by direct observation of the local density of states (LDOS) in scanning tunneling microscope experiments. ${ }^{11-13}$

For infinite 2D systems (e.g., graphene) the one-parameter scaling theory predicts electron localization to occur for arbitrary strengths of the Anderson disorder, ${ }^{15}$ with an exponentially large localization length for weak disorder. In finite 2D systems, e.g., in currently available graphene flakes or potential graphene nanodevices extended states may thus exist if the sample is smaller than the localization length. ${ }^{14}$

Early numerical work on localization concentrated on the calculation of the averaged localization length or the inverse participation ratio of single-particle wave functions. ${ }^{16,17}$ Quite generally investigations of disordered systems require the study of distributions of local quantities. This makes the application of statistical methods necessary ${ }^{1,18}$ to compute, for example, the distribution of squared wave-function amplitudes ${ }^{19}$ participation ratios,${ }^{20}$ or the LDOS. ${ }^{21-25}$ On the analytical side, great insight into disordered systems was gained by the random matrix theory. ${ }^{26}$ Here the supersymmetric nonlinear $\sigma$ model formalism ${ }^{27}$ allows one to establish a link between random matrices and Anderson-type disorder models. Thereby the localization properties of a system are traced back to global symmetry properties such as timereversal invariance. Using the transfer-matrix technique, the $\sigma$ model may be solved exactly for quasi-one-dimensional (1D) geometries. ${ }^{28}$ In higher dimensions, only approximate results were obtained so far, e.g., by employing perturbative renormalization group $^{29}$ or saddle-point-integration methods. ${ }^{30}$
In view of the predicated universality of the results of the random matrix theory it is worthwhile to apply unbiased numerical schemes to check the results and perhaps detect possible deviations. To extract meaningful results from numerical studies the data clearly need to be supplemented by a careful finite-size analysis. In this way, the scaling properties of the distribution of the squared wave-function amplitude were recently used to determine the multifractal spectrum at the Anderson transition. ${ }^{31}$

While in principle all local quantities are equally suited to describe localization properties in terms of their distribution, for practical use the LDOS seems to be the most favorable. This is due to its accessibility by means of highly efficient numerical computation schemes, such as the Kernel Polynomial Method (KPM). ${ }^{32}$ Within the KPM the LDOS of any lattice site can be calculated to, de facto, arbitrary precision without an explicit diagonalization of the Hamiltonian. A further advantage of the LDOS is the fact that this singleparticle quantity remains meaningful even in the presence of interactions ${ }^{33-38}$ and because of its direct measurability by scanning tunneling microscopy. ${ }^{8,11-13}$

In this paper we revisit the local distribution approach for the LDOS, propose further technical refinements of the $\mathrm{KPM}$, and comment on potential pitfalls concerning its application. In particular, we investigate the influence of the dimension and the coordination number of the underlying lattice on the LDOS distribution. The results are contrasted with the analytically predicted log-normal shape of the LDOS distribution. ${ }^{20}$

\section{MODEL AND METHOD}

We consider the Anderson model of disorder,

$$
H=-t \sum_{\langle i j\rangle}\left(c_{i}^{\dagger} c_{j}+\text { H.c. }\right)+\sum_{i} \epsilon_{i} c_{i}^{\dagger} c_{i},
$$

where the operators $c_{i}^{\dagger}\left(c_{i}\right)$ create (annihilate) an electron in a Wannier state centered at site $i$. In Eq. (1), $t$ denotes the 
nearest-neighbor transfer integral. Depending on the dimensionality $(D=2$ or $D=3)$ and lattice structure (e.g., honeycomb, hypercubic, triangular), the number of nearest neighbors varies from three to six. The on-site potentials $\epsilon_{i}$ are drawn from a uniform probability distribution (box distribution)

$$
p\left[\epsilon_{i}\right]=\frac{1}{\gamma} \theta\left(\frac{\gamma}{2}-\left|\epsilon_{i}\right|\right) .
$$

The parameter $\gamma$ measures the strength of the disorder. Each different sample corresponds to one particular realization $\left\{\epsilon_{i}\right\}$.

Following Anderson, ${ }^{1}$ we consider the LDOS at lattice site $i$,

$$
\rho_{i}(E)=\sum_{n=1}^{N}|\langle i \mid n\rangle|^{2} \delta\left(E-E_{n}\right),
$$

where $|i\rangle=c_{i}^{\dagger}|0\rangle$, and $|n\rangle$ is a single-electron eigenstate of $H$ with energy $E_{n}$, where $N$ denotes the number of sites and we assume periodic boundary conditions. The LDOS can be determined very efficiently by the KPM. The KPM is, in principle, an exact diagonalization (ED) approach, which is based on the expansion of the rescaled Hamiltonian into a finite series of Chebyshev polynomials. ${ }^{32,39}$ Calculating the expansion coefficients requires only sparse matrix-vector multiplications with $H$. In order to avoid Gibbs oscillations arising from the finite expansion order, the exact spectrum is convoluted with the strictly positive Jackson kernel. In consequence, each $\delta$-peak is approximated by an almost Gaussian of width $\sigma$, which depends on the order $M$ of the polynomial approximation. ${ }^{39}$ By definition, the Gaussian is reduced to $e^{-1 / 2} \approx 0.61$ of the peak value at distance $\sigma$. In the following, we will refer to $\sigma$ shortly as width of the Jackson kernel. Due to the peak broadening, the calculated LDOS at a given energy, $E_{t}$, also contains contributions from states with nearby energies (see Fig. 1). At first glance, this seems to be a drawback, preventing us from resolving the localization properties of a single state. However, the characteristics of one state for one realization of disorder are irrelevant due to the statistical nature of the Hamiltonian. Instead, an ensemble of disorder realizations has to be considered. In this respect, the finite energy resolution is even helpful since the exact eigenenergies vary from one random sample to the next. Hence the finite width of the Jackson kernel ensures the inclusion of at least some states with energies close to $E_{\mathrm{t}}$.

Determining the localization properties of a system for given parameters $(\gamma, E)$ by the local distribution approach is a two-step procedure. In a first step, we accumulate the probability distribution of the LDOS at energy $E, f\left[\rho_{i}(E)\right]$, within a histogram. Therefore, we probe many different lattice sites $i$ and realizations of disorder $\left\{\epsilon_{i}\right\}$. By normalizing the LDOS to its mean value $\rho_{\text {me }}(E)=\left\langle\rho_{i}(E)\right\rangle$, i.e., $\widetilde{\rho}_{i}=\rho_{i} / \rho_{\text {me }}$, we can pin down the characteristics of $f\left[\widetilde{\rho}_{i}(E)\right]$ in terms of its shape. For this we note that the distributions $f\left[\tilde{\rho}_{i}(E)\right]$ match with respect to norm and mean value for all $\gamma$ and $E$. Since $\widetilde{\rho}_{i}(E)$ varies over several orders of magnitude for a disordered system, a

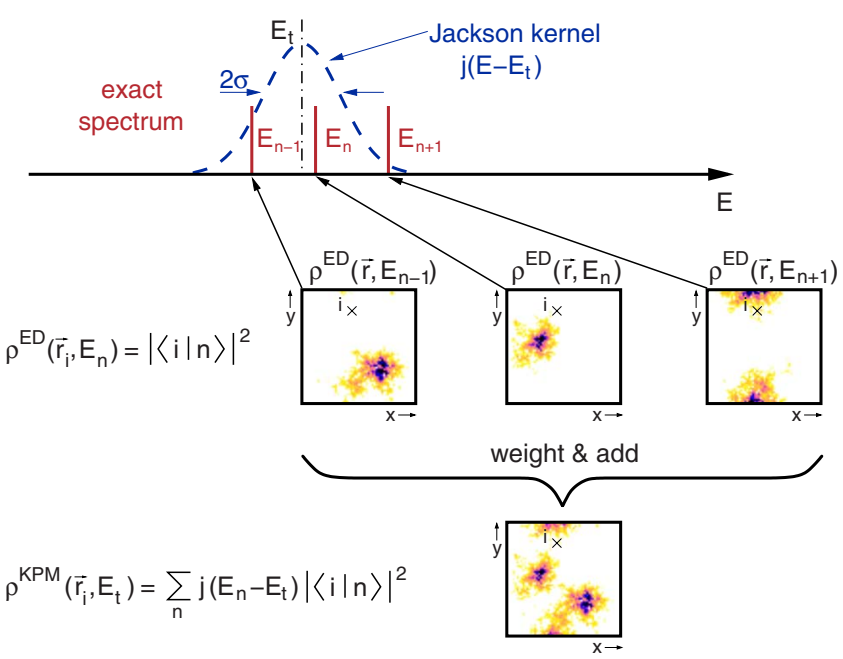

FIG. 1. (Color online) Relation between the LDOS as calculated by ED and KPM. For a single eigenstate $|n\rangle$, the LDOS at site $\vec{r}_{i}$ and energy $E_{n}$ is simply the squared amplitude of the wave function at this site, $\rho_{i}^{\mathrm{ED}}\left(E_{n}\right)=\rho^{\mathrm{ED}}\left(\vec{r}_{i}, E_{n}\right)=|\langle i \mid n\rangle|^{2}$. The squares schematically depict the LDOS values on all lattice sites for three different eigenstates, using the same color map as in Fig. 3. The cross indicates an arbitrary site $i$ of the sample. In contrast, the KPM assembles contributions from all eigenstates in the energetic vicinity of the target energy $E_{\mathrm{t}}$. Here the width $\sigma$ of the Jackson kernel $j\left(E-E_{\mathrm{t}}\right)$ determines both the number of involved states and their weight in the superposition, $\rho_{i}^{\mathrm{KPM}}\left(E_{\mathrm{t}}\right)=\sum_{n} j\left(E_{n}-E_{\mathrm{t}}\right)|\langle i \mid n\rangle|^{2}$.

logarithmic partitioning of the histogram bins is advantageous. Equivalently, we can consider the assembly of $\tilde{f}\left[\ln \widetilde{\rho}_{i}(E)\right]$, defined by the differentials

$$
f\left[\widetilde{\rho}_{i}(E)\right] d\left[\widetilde{\rho}_{i}(E)\right]=\tilde{f}\left[\ln \widetilde{\rho}_{i}(E)\right] d\left[\ln \widetilde{\rho}_{i}(E)\right] .
$$

In order to minimize the statistical fluctuations, the width of the histogram bins $\xi$ has to be adapted to the calculated number of LDOS values, $K$. Following Scott, ${ }^{40}$ we use $\xi$ $=3.49 \sigma_{0} K^{-1 / 3}$, where $\sigma_{0}$ is the width of a Gaussian fit to $\widetilde{f}\left[\ln \widetilde{\rho}_{i}(E)\right]$. The logarithmic representation, $\tilde{f}\left[\ln \widetilde{\rho}_{i}(E)\right]$, enables a single comparison with the suggested log-normal distribution, ${ }^{20}$

$$
\phi_{0}\left(\tilde{\rho}_{i}\right)=\frac{1}{\sqrt{2 \pi \sigma_{0}^{2}}} \frac{1}{\tilde{\rho}_{i}} \exp \left[-\frac{\left(\ln \tilde{\rho}_{i}-\mu\right)^{2}}{2 \sigma_{0}^{2}}\right] .
$$

We note that in transforming $f \rightarrow \tilde{f}$ a Jacobian appears, and the log-normal distribution on a linear scale acquires an additional factor $1 / \widetilde{\rho}_{i}(E)$ as compared to a Gaussian distribution on the logarithmic scale. In consideration of the fact that the mean value and the norm of $f\left[\tilde{\rho}_{i}(E)\right]$ are unity, the same should hold for the distribution $\phi_{0}\left(\widetilde{\rho}_{i}\right)[\mathrm{Eq} .(5)]$. This can be ensured by requiring $2 \mu=-\sigma_{0}^{2}$ in Eq. (5), thereby reducing the number of free fit-parameters to a single one. Due to this additional requirement, $\phi_{0}\left(\widetilde{\rho}_{i}\right)$ fulfills the symmetry relation

$$
\tilde{\rho}_{i}^{3} \phi_{0}\left(\widetilde{\rho}_{i}\right)=\phi_{0}\left(\widetilde{\rho}_{i}^{-1}\right),
$$

which was first derived for the nonlinear $\sigma$ model, ${ }^{41,42}$ and which was recently shown to lead to the prediction of a nontrivial symmetry satisfied by multifractal exponents. ${ }^{43}$ As any 


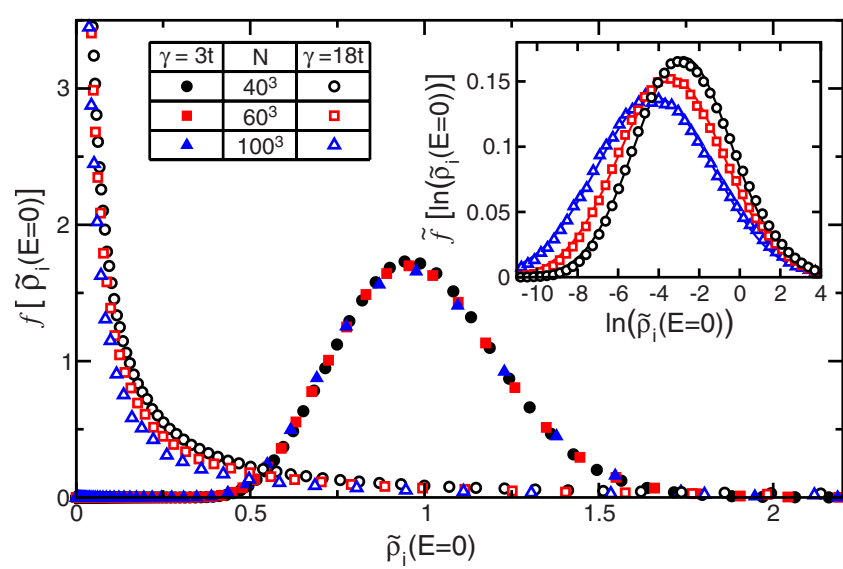

FIG. 2. (Color online) Probability distribution of the normalized LDOS for states in the band center of a $3 \mathrm{D}$ cubic lattice. To ensure a proper statistics, the LDOS values of $K=2 \times 10^{6}, 4 \times 10^{5}, 8$ $\times 10^{4}$ different sites and disorder realizations were calculated for $N=40^{3}, 60^{3}, 100^{3}$. The KPM resolution was adapted to contain $N_{k}$ $=140$ states within the kernel, irrespective of the system size. In the inset $\tilde{f}\left[\ln \tilde{\rho}_{i}\right]$ is given for the localized case $(\gamma=18 t)$ by symbols together with a log-normal fit to the data (solid lines).

microscopic disordered system close to the transition point is equivalent to the nonlinear $\sigma$ model, this relation should be valid also for Anderson model (1). A more detailed discussion of this issue is given in Appendix A.

The second decisive step of the local distribution approach is the finite-size scaling of the obtained energydependent LDOS distributions, which finally allows one to address the problem of Anderson localization. Keeping the number of states within the Jackson kernel, $N_{k}$, fixed, localized and extended states behave differently upon increasing $N$ : for extended states, the normalization of $\widetilde{\rho}_{i}$ to $\rho_{\text {me }}$ ensures that $f\left[\tilde{\rho}_{i}(E)\right]$ is independent of the system size. In contrast, despite fixed norm and mean, localized states are characterized by a shift of $f\left[\widetilde{\rho}_{i}(E)\right]$ toward zero as the system size increases. In the theoretical limit of infinite system size, the distribution will become singular at zero. Additional, more technical details of the LDOS approach are given in Appendix B.

\section{RESULTS AND DISCUSSION}

\section{A. Three-dimensional lattices}

\section{Cubic lattice}

In order to illustrate the power and reliability of the local distribution approach, let us first consider the threedimensional (3D) cubic lattice. Here extended states exist for $\gamma<\gamma_{c} \approx 16.5 t$, whereas for $\gamma>\gamma_{c}$ all states are localized. ${ }^{4}$ In Fig. 2 the different scaling behaviors of $f\left[\widetilde{\rho}_{i}(E=0)\right]$ with increasing $N$ can be readily observed: (i) for weak disorder $(\gamma=3 t)$ the distribution is completely unaffected upon increasing the system size from $N=40^{3}$ to $N=100^{3}$. The almost Gaussian shape of $f\left[\widetilde{\rho}_{i}(E=0)\right]$ reflects the random LDOS fluctuations from one site to another and the absence of any superimposed global structure. Note that the $N$-independent width of the distribution (between $50 \%$ and $150 \%$ of $\rho_{\mathrm{me}}$ ) is due the fixed $N_{k}$ scaling and its normalization to $\rho_{\text {me }}$. Of course, concerning absolute values it narrows with increasing $N$ because of the normalization of the individual eigenstates. (ii) For strong disorder $(\gamma=18 t)$ the distribution markedly depends on $N$. Its shape distinctly differs from a Gaussian distribution and closely resembles a log-normal distribution (see inset of Fig. 2). The maximum shifts by an order of magnitude when going from $N=40^{3}$ to $N=100^{3}$. The LDOS data and their log-normal fit are seen to match perfectly over several orders of magnitude. Here it should be emphasized that a distinction between localized and extended states solely based on the shape of the distribution at fixed system size is not meaningful—only the behavior of the distribution upon finite-size scaling is relevant. ${ }^{44}$ It is known that the distribution of the LDOS of extended states can be described equally well by a log-normal distribution. Both distributions agree for lattices without disorder, and are hardly distinguishable as long as mean and most probable value do not differ significantly.

\section{Honeycomb lattice}

In addition to the discussion of the LDOS distribution, we now show how the spatial structure of the LDOS differs for a typical, but particular exact eigenstate of a certain sample in the extended and localized regime, respectively (see Fig. 3 ). To this end we switch from the 3D cubic lattice to a $3 \mathrm{D}$ honeycomb lattice sketched in the left panel of Fig. 3, whereby the number of nearest-neighbor sites is reduced from six to five. The unperturbed bandwidth for the 3D honeycomb lattice without Anderson disorder is $W_{0}=10 t$ instead of $W_{0}=12 t$ for the cubic one. In order to figure out how the same disorder strength influences the localization properties for different coordination numbers, we have to compare results for the same ratio of $\gamma / W_{0}$ rather than those for absolute $\gamma / t$ values. To make contact with results shown in Fig. 2, we therefore use $\gamma=2.5 t=0.25 W_{0}$ and $\gamma=15 t=1.5 W_{0}$ in order to model the weak and strong disorder case, respectively. Both for extended and localized states we recover the above quoted characteristics. Namely, for weak disorder we find that the LDOS spreads over the whole lattice, showing moderate amplitude fluctuations without a global structure (left panel of Fig. 3). For strong disorder there occurs a concentration of the LDOS on a spatially restricted, filamentary subset of all lattice sites (right panel of Fig. 3). However, since Fig. 3 shows particular eigenstates, the LDOS variations are more pronounced than for the superposed KPM data (cf. Fig. 8 in Appendix B). We note that the finite-size scaling of $f\left[\widetilde{\rho}_{i}(E=0)\right]$ for the 3D honeycomb lattice (not shown) qualitatively agrees with the one presented in Fig. 2. Quantitatively, for the heavily disordered 3D honeycomb lattice, $\widetilde{f}\left[\ln \widetilde{\rho}_{i}(E=0)\right]$ is concentrated at smaller values for all $N$, indicating a stronger localization, i.e., a smaller localization length. Despite the similarity of this lattice to graphite, our results may not be transferred directly to this system. For graphite, the layer stacking differs (Bernal stacking), and the interlayer coupling is much weaker than the intralayer one. 

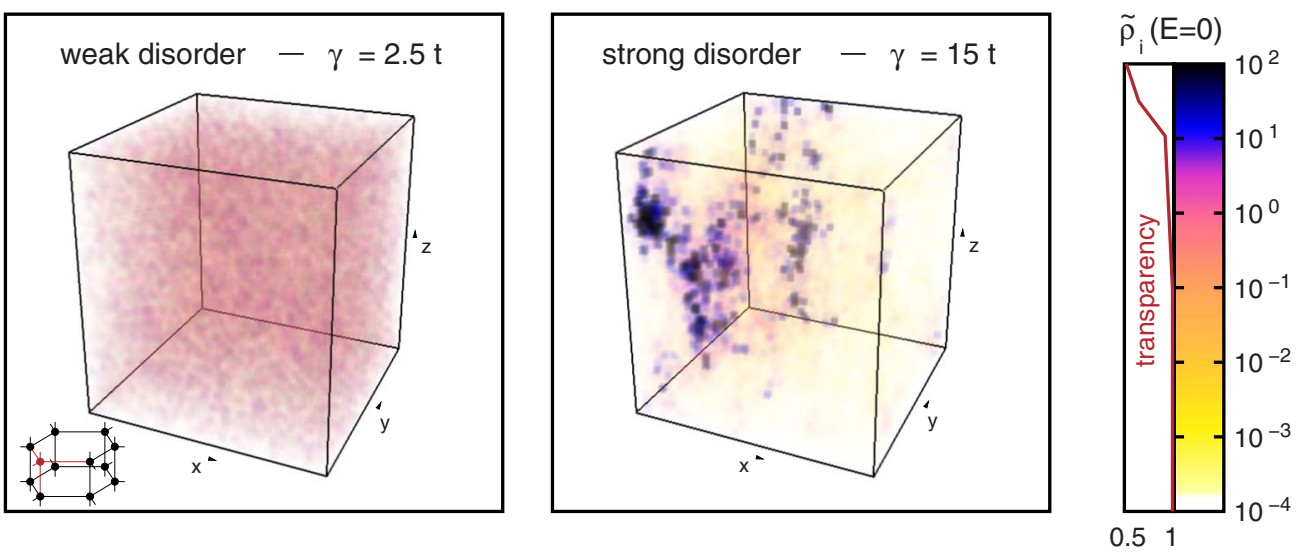

FIG. 3. (Color online) Spatially resolved LDOS of the eigenstate with energy closest to $E=0$ for a particular disorder realization of the Anderson model. We contrast the results obtained for weak disorder $(\gamma=2.5 t)$, where the particle is spread over the whole sample (left-hand panel), with those for strong disorder $(\gamma=15 t)$, where the particle is confined to a finite region (right-hand panel). Data were obtained by ED for the 3D honeycomb lattice with $N=32^{3}$ sites. Each lattice point is represented by a small cube, with color corresponding to the squared amplitude of the wave function. In order to permit a view into the crystal, in addition we used the transparency gradient given on the left of the color bar.

\section{B. Two-dimensional lattices}

\section{Square lattice}

We now turn to the $2 \mathrm{D}$ case and compare the distribution of the LDOS for the 3D cubic lattice with that for a $2 \mathrm{D}$ square lattice (see Fig. 4). As predicted by the one-parameter scaling theory, ${ }^{15}$ arbitrarily weak disorder will cause localization in 2D. However, the localization length can become so large that the localized nature of the states can be easily missed within a numerical approach. Investigating a system


FIG. 4. (Color online) Comparison of $\tilde{f}\left[\ln \left(\widetilde{\rho}_{i}(E=0)\right)\right]$ for $2 \mathrm{D}$ square and $3 \mathrm{D}$ cubic lattices. In both cases $\gamma=0.25 W_{0}$ and $\gamma$ $=1.5 W_{0}$. We used $N_{k}=140$ for all $N$ and assembled $K=2 \times 10^{6}, 4$ $\times 10^{5}, 8 \times 10^{4}$ LDOS values for $N=40^{3}, 60^{3}, 100^{3}$ and $200^{2}, 400^{2}, 800^{2}$. The solid lines give fits to log-normal distributions. with a localization length (much) larger than the considered system size gives us the opportunity to demonstrate the power of our method. Again, we compare constant ratios $\gamma / W_{0}=0.25$ and $\gamma / W_{0}=1.5$, respectively, where the bandwidth for the 2D square lattice without disorder is $W_{0}=8 t$. For strong disorder (upper panels in Fig. 4), the more pronounced shifting of $\widetilde{f}\left[\ln \left(\widetilde{\rho}_{i}(E=0)\right)\right]$ indicates the even stronger localization in 2D. To speak of localization lengths is, however, dangerous due to the ambiguous base for the finitesize scaling. While the spectral properties are governed by the Hilbert-space dimension $N$, the localization length has to be compared to the linear size of the system. Quantitative statements about localization lengths are therefore limited to systems of equal dimensionality. The true challenge for any method is, of course, the weak disorder case (lower panels in Fig. 4), where an extended state (3D) has to be distinguished from a localized one with large localization length (2D). As already shown in Fig. 2 on a linear scale, the 3D data show no dependence on $N$ at all on a double-logarithmic scale, spanning several orders of magnitude. By contrast, the $\mathrm{N}$-dependence of the 2D data is obvious. Although we do not see the pronounced shift of the maximum of the distribution as in the strong disorder case, we observe a systematic broadening of the distribution. Due to the constant norm and mean, this broadening can be directly related to a shift of the maximum despite its faint visibility. By this, we may detect localized behavior even if the localization length distinctly exceeds the system size. A word of caution has to be added concerning the tails of the numerically assembled distribution. In an ensemble of $K$ LDOS values we cannot reliably expect to find events outside the range $\xi \widetilde{f}\left[\ln \widetilde{\rho}_{i}\right] \geq 1 / K$ because of their low probability. The prominent role of the distribution tails calls for maximizing $K$ to extend their range of reliability. Comparing the LDOS distributions to the corresponding log-normal fits, the agreement is again remarkable. 


\section{Triangular and honeycomb lattices}

Finally we apply the local distribution approach to other 2D lattices and address the influence of the coordination number on the localization properties. To this end we study the triangular and the honeycomb lattices. In view of the current intense studies of graphene, the latter is of great experimental and theoretical relevance. ${ }^{10,45}$ Calculating $\rho_{i}(E$ $=0$ ) for weakly disordered graphene requires additional care due to the specifics of the electron dispersion at and near the Dirac point. For the ordered case we have $\rho_{\text {me }} \sim|E|$. At finite $N$, with appropriate linear crystal size, four states exist at $E$ $=0$ surrounded by a finite-size gap and further stray states. Evaluating $\rho_{\text {me }}$ by means of the KPM, this value at $E=0$ will depend crucially on $\sigma$, i.e., on the broadening of the vicinal peaks. Furthermore, $\rho_{\text {me }}$ varies significantly on the scale of the kernel width and can no longer be treated as constant when calculating $N_{k}$. As a consequence, the correct value of $N_{k}$ has to be calculated self-consistently using (B1) prior to the assembly of the LDOS histogram data. According to the bipartite graphene lattice structure with three nearest neighbors the bandwidth for the honeycomb lattice is $W_{0}=6 t$. Since the triangular lattice is nonbipartite, $\rho_{\text {me }}$ is asymmetric with band edges in the ordered case at $E=-6 t$ and $E=3 t$. Despite the six nearest neighbors of the triangular lattice, the electronic bandwidth is $W_{0}=9 t$ only, in contrast to the $3 \mathrm{D}$ cubic lattice with $W_{0}=12 t$. In Fig. 5 we compare how weak $\left(\gamma=0.25 W_{0}\right)$ and moderate $\left(\gamma=0.625 W_{0}\right)$ disorder influence electronic states on the above lattices. In all six cases we observe clear indications for localization since $\tilde{f}\left[\ln \left(\widetilde{\rho}_{i}(E\right.\right.$ $=0)$ )] either visibly shifts on increasing $N$ (right column) or can be deduced from the systematic tail broadening (left column). The agreement with the corresponding log-normal fits also holds equally well for all lattice types and both disorder strengths. The strongest localization effects, i.e., the most pronounced shifting of $\widetilde{f}\left[\ln \left(\widetilde{\rho}_{i}(E=0)\right)\right]$ can be observed for the honeycomb lattice (see right column of Fig. 5). We attribute this to the smallest coordination number, a conclusion that is supported by the less pronounced but clearly observable localization for the triangular lattice.

\section{CONCLUSIONS}

By performing extensive, numerically exact calculations of the distribution of the local density of states (LDOS) of disordered electrons on various two- and three-dimensional lattices we showed that the local distribution approach can reliably distinguish between localized and extended states. A careful finite-size scaling analysis is crucial for the success of this method. Localized states are identified by the broadening of the LDOS distribution and its shift to zero for increasing system size. Remarkably, a log-normal distribution perfectly fits the LDOS data over up to ten orders of magnitude, depending on the dimensionality, lattice structure and disorder strength, with only minor systematic deviations for the outer tails of the distribution. Close to the Andersontransition point the LDOS distribution corroborates a symmetry relation originally found for the nonlinear $\sigma$ model. In contrast to localized states, the LDOS distribution for extended states is system-size independent.
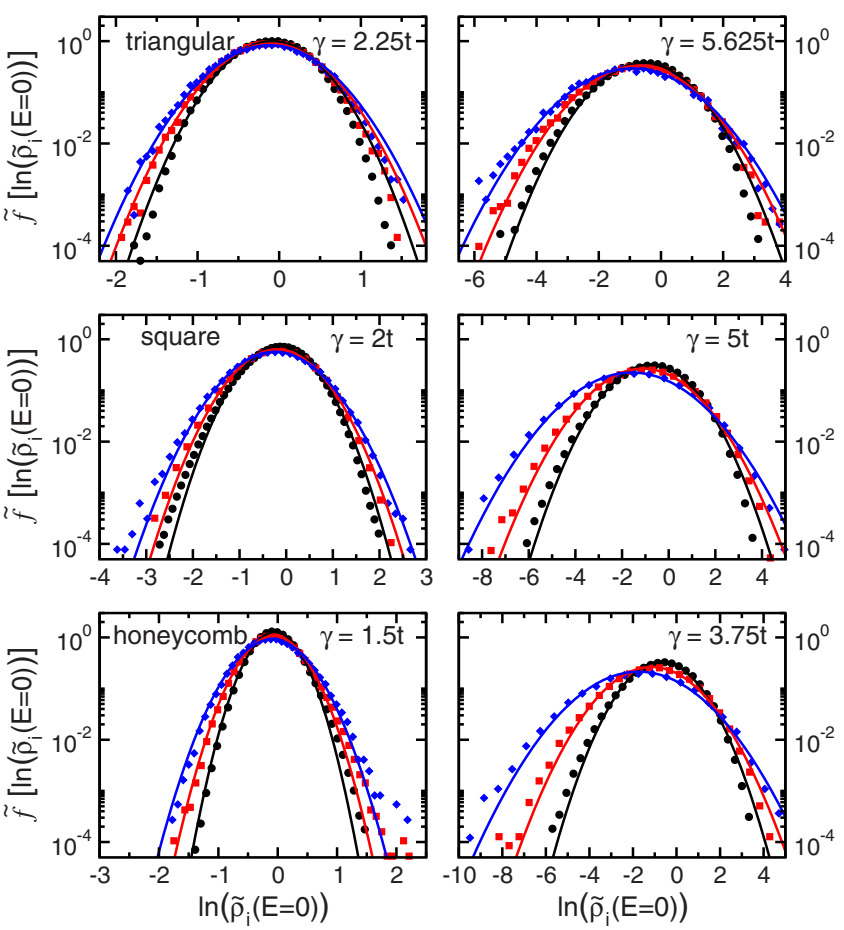

FIG. 5. (Color online) Comparison of $\tilde{f}\left[\ln \left(\widetilde{\rho}_{i}(E=0)\right)\right]$ for different 2D lattices. We consider the cases of weak $\left(\gamma=0.25 W_{0}\right)$ and moderate $\left(\gamma=0.625 W_{0}\right)$ disorder, with $N_{k}=140$. For the triangular and square lattices the same symbols as in Fig. 4 for $N$ $=200^{2}, 400^{2}, 800^{2}$ are used. For the honeycomb lattice we studied $N=192^{2}, 396^{2}, 792^{2}$ to match the correct boundary conditions and assembled $K=2 \times 10^{6}, 4 \times 10^{5}, 8 \times 10^{4}$ LDOS values (from small to large $N$ ).

The stability of the Chebyshev expansion permits a numerically exact calculation of the LDOS by means of the Kernel Polynomial Method, the accuracy being limited only by machine precision. Special care is required when calculating the LDOS at energies where the mean DOS strongly varies. Here one has to guarantee that the number of states within the Jackson kernel is always kept constant in the finite-size scaling. The symmetry relation of the LDOS can be used to improve the data analysis for Anderson disordered systems.

Our numerically exact results confirm the existence of a localization threshold in 3D as first predicted by Anderson, ${ }^{1}$ as well as localization for any strength of disorder ${ }^{15}$ in $2 \mathrm{D}$. By increasing the coordination number of the lattice the influence of disorder is reduced, such that comparable disorder strengths evoke the shortest localization lengths for the graphene lattice. Considering finite 2D, or quasi-1D graphene nanoribbons, the localization length may nevertheless easily exceed the size of potential nanodevices. In addition, boundary effects will become important. As a result, charge transport will become possible despite the existence of actually localized wave functions. Concerning future investigations of the complex interplay between disorder and electron-electron ${ }^{2,46,47}$ or electron-phonon interaction ${ }^{23}$ in finite, low-dimensional systems the LDOS distribution approach shows great promise. 


\section{ACKNOWLEDGMENTS}

We thank A. Alvermann, F. X. Bronold, B. Shapiro, A. Weiße, G. Wellein, and P. Wölfle for stimulating discussions. This work was funded by the Competence Network for Technical/Scientific High-Performance Computing in Bavaria (KONWIHR II) and was supported by the TRR 80 and SPP 1459 of the Deutsche Forschungsgemeinschaft. The numerical calculations were performed on the HLRBII, Leibniz Supercomputing Center Munich, and on the tera-flop compute cluster at the Institute of Physics, Greifswald University and Regional Computing Center Erlangen.

\section{APPENDIX A: SYMMETRY RELATION (6)}

Log-normal distribution (5) fulfills symmetry relation (6) only if $2 \mu=\sigma^{2}$. Then, Eq. (6) can be proven by simply completing the square in the exponent,

$$
\begin{aligned}
\tilde{\rho}_{i}^{3} \phi_{0}\left(\widetilde{\rho}_{i}\right) & =\frac{1}{\sqrt{2 \pi \sigma_{0}^{2}}} \widetilde{\rho}_{i}^{2} \exp \left[-\frac{\left(\ln \tilde{\rho}_{i}-\mu\right)^{2}}{2 \sigma_{0}^{2}}\right] \\
& =\frac{1}{\sqrt{2 \pi \sigma_{0}^{2}}} \widetilde{\rho}_{i} \exp \left[-\frac{\left(\ln \tilde{\rho}_{i}-\mu\right)^{2}}{2 \sigma_{0}^{2}}+\ln \widetilde{\rho}_{i}\right] \\
& =\frac{1}{\sqrt{2 \pi \sigma_{0}^{2}}} \tilde{\rho}_{i} \exp \left[-\frac{\left(-\ln \widetilde{\rho}_{i}-\mu\right)^{2}}{2 \sigma_{0}^{2}}\right]=\phi_{0}\left(\tilde{\rho}_{i}^{-1}\right) .
\end{aligned}
$$

For any log-normal distribution that violates $2 \mu=\sigma^{2}$, the above procedure maps $\phi_{0}\left(\widetilde{\rho}_{i}\right)$ onto a different log-normal distribution, which is shifted and rescaled. A more intuitive understanding of this symmetry property can be obtained by discussing it in terms of $x=\ln \tilde{\rho}_{i}$ instead of $\tilde{\rho}_{i}$. On a logarithmic scale, Eq. (6) reads as

$$
e^{x} \widetilde{\phi}_{0}(x)=\widetilde{\phi}_{0}(-x),
$$

where $\widetilde{\phi}_{0}(x)$ is a normal distribution. Incorporating the $e^{x}$ factor into the exponential of $\widetilde{\phi}_{0}(x)$, the position of the maximum is shifted and the term that occurs by completing the square gives an additional scaling factor. For illustration consider the log-normal distributions

$$
\widetilde{g}_{ \pm}(x)=\frac{1}{\sqrt{2 \pi \sigma_{0}^{2}}} \frac{1}{1+e^{\mp \alpha \sigma_{0}^{2}}} \exp \left[-\frac{1}{2 \sigma_{0}^{2}}\left(x+\frac{\sigma_{0}^{2}}{2} \pm \alpha \sigma_{0}^{2}\right)^{2}\right] \text {, }
$$

for which $e^{x} \widetilde{g}_{ \pm}(x)=\widetilde{g}_{\mp}(-x)$. As a result, their sum

$$
\widetilde{\phi}_{2}(x)=\widetilde{g}_{+}+\widetilde{g}_{-}
$$

is invariant with respect to Eq. (A2). The additional parameter $\alpha$ can be used in order to improve the quality of the fit since the different prefactors of the Gaussians in $\widetilde{\phi}_{2}(x)$ also allow for an adjustment to asymmetric data. Figure 6 contrasts the best fits of our LDOS data to $\widetilde{\phi}_{0}(x)$ and $\widetilde{\phi}_{2}(x)$. Obviously, the improvement which can be achieved by the more complicated $\widetilde{\phi}_{2}(x)$ fit is not very significant. Therefore, we restricted ourselves in the main part of the paper to fits using $\widetilde{\phi}_{0}(x)$.

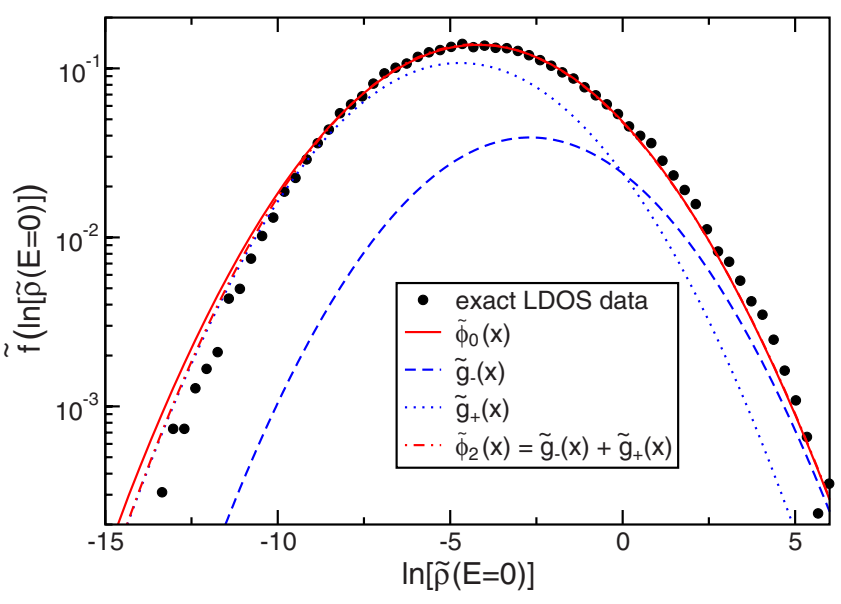

FIG. 6. (Color online) Comparison of the quality of various fits to our LDOS data including the more complicated fit function, $\widetilde{\phi}_{2}(x)$, Eq. (A4), comprising a sum of two log-normal distributions $g_{ \pm}(x)$. Upon symmetry operation (A2) the function $g_{+}(x)$ is mapped to $g_{-}(x)$ and vice versa. Note that $\widetilde{\phi}_{0}(x)=\widetilde{\phi}_{2}(\alpha=0, x)$. The LDOS data are taken from Fig. 2 and correspond to $\gamma=18 t, N=100^{3}$.

The variation of the LDOS data over several orders of magnitude makes it numerically challenging to single out an optimal log-normal fit. In particular, it is not easy to judge its quality by the eye. Throughout the paper we employ the nonlinear Levenberg-Marquardt algorithm. ${ }^{48}$ Thereby we used the pristine data without any additional weighting (which might be introduced to accentuate specific data regions, e.g., the distribution tails). A well established graphical technique for testing the agreement of a given data set with a (log-)normal distribution is the (log-)normal probability plot. ${ }^{49}$ Here the cumulative distribution function $(\mathrm{CDF})$ is plotted and the ordinate is scaled according to the inverse of the cumulative (log-)normal distribution function. Then a (log-)normal distribution will show up as a straight line (see upper left panel of Fig. 7). This presentation underlines the almost perfect agreement of our LDOS data with a (log)normal distribution over the whole data range. Deviations for large values of $\tilde{\rho}_{i}$ are due to the poor statistics of those rare events in our finite ensemble of realizations.

We see that, by exploiting symmetry (6) [or equivalently Eq. (A2)], any data analysis for disordered systems may profit from the statistically more settled data near the maximum of the distribution in order to enlarge the reliability of the data for very large values of $\rho_{i}$ (see other panels of Fig. 7). Self-evidently the symmetry should be used in this direction only. Mirroring the statistically poor data from the distribution tails to regions near the distribution maximum is unprofitable. Nevertheless, such a crosscheck offers the possibility to test the reliability of the numerical data, where deviations may be attributed to bad statistics $(K)$, finite-size effects $\left(N, N_{\mathrm{k}}\right)$, or the fact that we are too far away from the Anderson-transition point $\left(\gamma-\gamma_{c}\right)$. To illustrate the latter aspect, we determined the LDOS distribution at $\gamma=16.5 t \simeq \gamma_{c}$, yielding - as compared to $\gamma=18 t$ (for the same $\left(K, N, N_{k}\right)$ - a better agreement with symmetry relation (A2). Increasing $N$ clearly reduces the finite-size effects. Therefore the data presented for $\gamma=18 t, N=100^{3}$ are more reliable than those for 


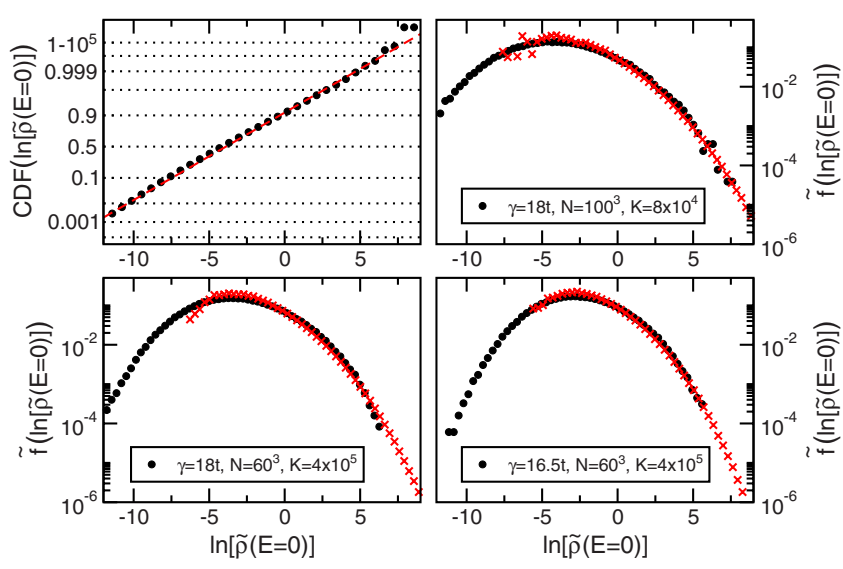

FIG. 7. (Color online) Upper left panel: log-normal probability plot for the LDOS distribution for $\gamma=18 t, N=100^{3}$ taken from Fig. 2. Only each second data point is depicted by a filled circle and compared with the (red dashed) straight line. Other panels: illustration to which extent symmetry relation (A2) holds for the exact LDOS data for different system sizes, number of realization, and disorder strengths. Calculated LDOS data are shown as filled circles while crosses give the results obtained by additionally exploiting symmetry relation (6). The data in all panels were calculated using $N_{k}=140$.

$N=60^{3}$ despite the fewer number of realizations $K$ in the latter case.

\section{APPENDIX B: ADAPTION OF THE KPM RESOLUTION}

The resolution of the original KPM is not uniform in the whole energy interval. For fixed expansion order the Jackson kernel becomes narrower the closer it is located near the edges of the spectrum. In order to allow for a clear distinction between resolution and localization effects, a refinement of the KPM has been proposed in Ref. 50, ensuring constant energy resolution over the whole spectrum. Still, this refinement does not account for one aspect that might become important for systems with a strongly varying DOS, such as graphene: if the DOS (and therefore the mean level spacing) varies noticeably throughout the spectrum, an adjustment of the kernel width $\sigma$ to $\rho_{\text {me }}$ is required. Thus, by adapting $\sigma$, the same number of states within the Jackson kernel

$$
N_{k}\left(E_{\mathrm{t}}\right)=N \int_{E_{\mathrm{t}}-\sigma\left(E_{\mathrm{t}}\right)}^{E_{\mathrm{t}}+\sigma\left(E_{\mathrm{t}}\right)} \rho_{\mathrm{me}}(E) d E
$$

is kept constant during the finite-size scaling for all energies $E_{\mathrm{t}}$. In many cases $\rho_{\text {me }}$ is approximately constant in the relevant energy interval and $N_{k} \simeq 2 \sigma N \rho_{\text {me }}$. However, near the Dirac point in graphene the vanishing DOS requires a selfconsistent evaluation of Eq. (B1). Initial calculations of $\rho_{\text {me }}$ are performed most efficiently by using delocalized random vectors in the KPM. ${ }^{32}$ This implicit averaging drastically improves the statistical convergence of $\rho_{\text {me }}$ but is afterwards unfeasible for the LDOS calculation itself.

A priori, there is no seeded value for $N_{k}$ to be used in the finite-size scaling. Only the limiting requirement of having some states within the kernel for each realization of disorder
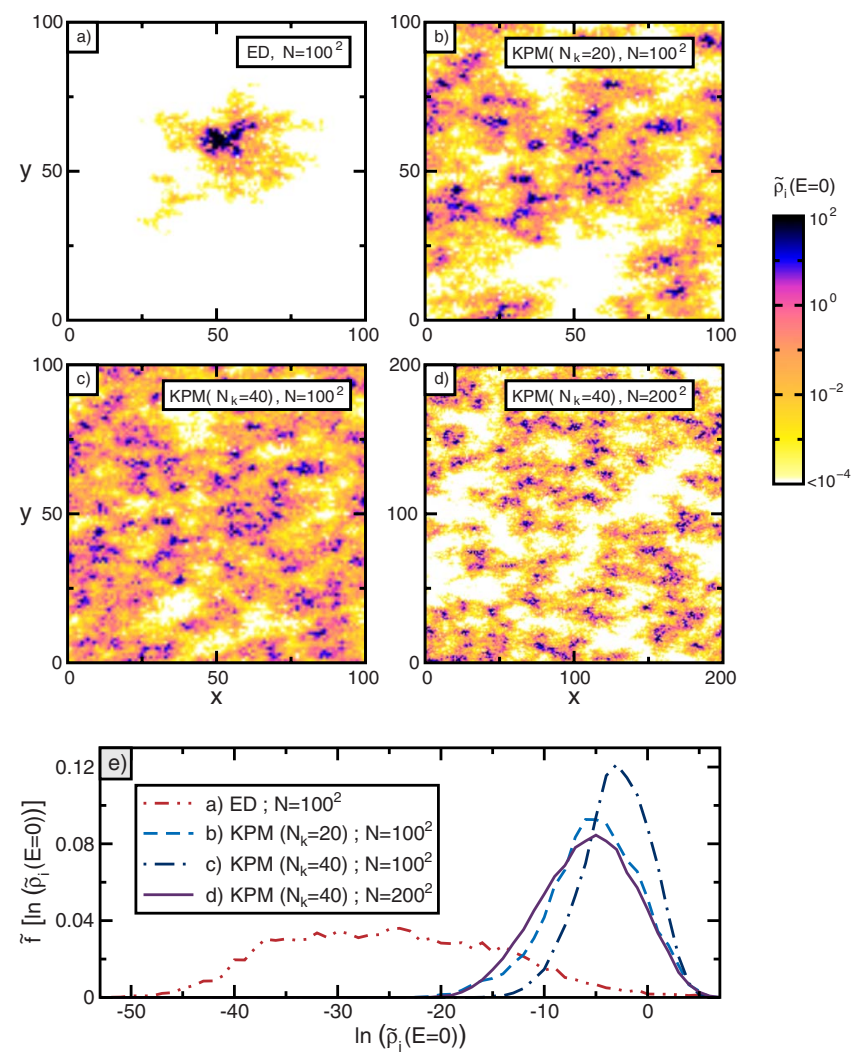

FIG. 8. (Color online) Influence of KPM resolution and system size on the LDOS and its distribution. Panel (a): LDOS of a single eigenstate in the band center for one realization of a strongly disordered $(\gamma=9 t)$ graphene lattice with $N=100^{2}$ sites. Panels (b) and (c): LDOS for the same realization calculated by KPM with different resolutions $\left(N_{k}=20,40\right)$. Panel (d): LDOS for a larger system $\left(N=200^{2}\right)$ at the same disorder strength and adapted KPM resolution such that $N_{k}=40$ is kept constant. Panel (e): corresponding probability distributions of the (normalized) LDOS, $\tilde{f}\left[\ln \left(\widetilde{\rho}_{i}(E\right.\right.$ $=0))$.

has to be fulfilled. In practice, one aims for a reasonable compromise between computational costs (CPU time $\sim M$ $\left.\sim 1 / \sigma \sim 1 / N_{k}\right)$ and energy resolution. The concrete choice of $N_{k}$ has a significant impact on the LDOS and its distribution (see Fig. 8). In the strongly localized regime individual eigenstates are confined to a tiny fraction of all lattice sites [Fig. 8(a)]. Eigenstates with similar energies are localized at different positions in space. Due to the finite width of the Jackson kernel, the LDOS obtained by KPM contains contributions of several of these states, depending on $N_{k}$ [Figs. 8(b) and 8(c)]. From Fig. 8(c) it can be seen that a too large value of $N_{k}$ masks the decay of the individual eigenstates since $\widetilde{\rho}_{i}(E)$ becomes comparable for most lattice sites. Accessing the localization properties is, however, possible by calculating the LDOS for a larger system using the same $N_{k}$ [Fig. 8(d)] and comparing both LDOS distributions [Fig. $8(\mathrm{e})]$. A shift of the LDOS distribution indicates Anderson localization.

Essentially, the properties of $\tilde{f}\left[\ln \left(\widetilde{\rho}_{i}\right)\right]$ are governed by the interplay of three parameters: the localization length $\lambda$, the 
system size $N$, and the number of states within the Jackson kernel $N_{k}$. Therefore, one might think of keeping $N$ fixed and investigate the behavior of $\tilde{f}\left[\ln \left(\widetilde{\rho}_{i}\right)\right]$ in dependence on $N_{k}$ [curves corresponding to Figs. 8(c) and 8(b)]. We use our initially proposed finite-size scaling [Fig. 8(c) vs Fig. 8(d)] since there the relevant ratio $\lambda / N$ is varied and $N_{k}$ only en- ters as a parameter. In comparison to the LDOS distribution from ED [curve corresponding to Fig. 8(a)], the LDOS distributions obtained by KPM have the numerical advantage of a reduced width [see Fig. 8(e)]. In consequence, limitations by machine precision will be less restrictive for the KPM but might become an issue in $\mathrm{ED}$ for $\lambda \ll N^{1 / D}$.
${ }^{1}$ P. W. Anderson, Phys. Rev. 109, 1492 (1958).

${ }^{2}$ P. A. Lee and T. V. Ramakrishnan, Rev. Mod. Phys. 57, 287 (1985).

${ }^{3}$ D. Vollhardt and P. Wölfle, in Electronic Phase Transitions, edited by W. Hanke and Y. V. Kopaev (North Holland, Amsterdam, 1992), p. 1.

${ }^{4}$ B. Kramer and A. Mac Kinnon, Rep. Prog. Phys. 56, 1469 (1993).

${ }^{5}$ F. Evers and A. D. Mirlin, Rev. Mod. Phys. 80, 1355 (2008).

${ }^{6}$ A. Lagendijk, B. van Tiggelen, and D. S. Wiersma, Phys. Today 62 (8), 24 (2009).

${ }^{7}$ A. Aspect and M. Inguscio, Phys. Today 62 (8), 30 (2009).

${ }^{8}$ A. Richardella, P. Roushan, S. Mack, B. Zhou, D. A. Huse, D. D. Awschalom, and A. Yazdani, Science 327, 665 (2010).

${ }^{9}$ L. Sanchez-Palencia and M. Lewenstein, Nat. Phys. 6, 87 (2010).

${ }^{10}$ K. S. Novoselov, A. K. Geim, S. V. Morozov, D. Jiang, Y. Zhang, S. V. Dubonos, I. V. Grigorieva, and A. A. Firsov, Science 306, 666 (2004).

${ }^{11}$ Y. Niimi, H. Kambara, and H. Fukuyama, Phys. Rev. Lett. 102, 026803 (2009).

${ }^{12}$ Y. Niimi, H. Kambara, T. Matsui, D. Yoshioka, and H. Fukuyama, Phys. Rev. Lett. 97, 236804 (2006).

${ }^{13}$ M. Morgenstern, J. Klijn, C. Meyer, and R. Wiesendanger, Phys. Rev. Lett. 90, 056804 (2003).

${ }^{14}$ G. Schubert, J. Schleede, and H. Fehske, Phys. Rev. B 79, 235116 (2009).

${ }^{15}$ E. Abrahams, P. W. Anderson, D. C. Licciardello, and T. V. Ramakrishnan, Phys. Rev. Lett. 42, 673 (1979).

${ }^{16}$ B. J. Last and D. J. Thouless, J. Phys. C 7, 699 (1974).

${ }^{17}$ F. Wegner, Z. Phys. B 36, 209 (1980).

${ }^{18}$ R. Abou-Chacra, D. J. Thouless, and P. W. Anderson, J. Phys. C 6, 1734 (1973).

${ }^{19}$ V. I. Fal'ko and K. B. Efetov, Phys. Rev. B 52, 17413 (1995).

${ }^{20}$ A. D. Mirlin, Phys. Rep. 326, 259 (2000).

${ }^{21}$ A. D. Mirlin and Y. V. Fyodorov, Phys. Rev. Lett. 72, 526 (1994).

${ }^{22}$ A. D. Mirlin, Phys. Rev. B 53, 1186 (1996).

${ }^{23}$ F. X. Bronold, A. Alvermann, and H. Fehske, Philos. Mag. 84, 673 (2004).

${ }^{24}$ A. Alvermann and H. Fehske, Eur. Phys. J. B 48, 295 (2005).

${ }^{25}$ A. Alvermann and H. Fehske, Lect. Notes Phys. 739, 505 (2008).

${ }^{26}$ M. L. Mehta, Random Matrices (Academic Press, Boston, 1991).

${ }^{27}$ K. B. Efetov, Adv. Phys. 32, 53 (1983).
${ }^{28}$ Y. V. Fyodorov and A. D. Mirlin, Phys. Rev. Lett. 71, 412 (1993).

${ }^{29}$ B. L. Altshuler, V. E. Kravtsov, and I. V. Lerner, in Mesoscopic Phenomena in Solids, edited by B. L. Altshuler, P. A. Lee, and R. A. Webb (North-Holland, Amsterdam, 1991), p. 449.

${ }^{30}$ B. A. Muzykantskii and D. E. Khmelnitskii, Phys. Rev. B 51, 5480 (1995).

${ }^{31}$ A. Rodriguez, L. J. Vasquez, and R. A. Römer, Phys. Rev. Lett. 102, 106406 (2009).

${ }^{32}$ A. Weiße, G. Wellein, A. Alvermann, and H. Fehske, Rev. Mod. Phys. 78, 275 (2006).

${ }^{33}$ V. Dobrosavljević and G. Kotliar, Phys. Rev. Lett. 78, 3943 (1997); Philos. Trans. R. Soc. London, Ser. A 356, 57 (1998).

${ }^{34}$ F. X. Bronold and H. Fehske, Phys. Rev. B 66, 073102 (2002).

${ }^{35}$ V. Dobrosavljević, A. A. Pastor, and B. K. Nikolić, Europhys. Lett. 62, 76 (2003)

${ }^{36}$ K. Byczuk, W. Hofstetter, and D. Vollhardt, Phys. Rev. Lett. 94, 056404 (2005); 102, 146403 (2009).

${ }^{37}$ Yun Song, R. Wortis, and W. A. Atkinson, Phys. Rev. B 77, 054202 (2008).

${ }^{38}$ K. Byczuk, W. Hofstetter, and D. Vollhardt, in Fifty Years of Anderson Localization, edited by E. Abrahams (World Scientific, Singapore, 2010).

${ }^{39}$ A. Weiße and H. Fehske, Lect. Notes Phys. 739, 545 (2008).

${ }^{40}$ D. W. Scott, Biometrika 66, 605 (1979).

${ }^{41}$ A. D. Mirlin and Y. V. Fyodorov, J. Phys. I 4, 655 (1994).

${ }^{42}$ D. V. Savin, H.-J. Sommers, and Y. V. Fyodorov, JETP Lett. 82, 544 (2005).

${ }^{43}$ A. D. Mirlin, Y. V. Fyodorov, A. Mildenberger, and F. Evers, Phys. Rev. Lett. 97, 046803 (2006).

${ }^{44} \mathrm{G}$. Schubert, A. Weiße, G. Wellein, and H. Fehske, in High performance Computing in Science and Engineering, Garching 2004, edited by A. Bode and F. Durst (Springer-Verlag, Heidelberg, 2005), pp. 237-249.

${ }^{45}$ A. H. Castro Neto, F. Guinea, N. M. R. Peres, K. S. Novoselov, and A. K. Geim, Rev. Mod. Phys. 81, 109 (2009).

${ }^{46}$ D. Belitz and T. R. Kirkpatrick, Rev. Mod. Phys. 66, 261 (1994).

${ }^{47}$ S. V. Kravchenko and M. P. Sarachik, Rep. Prog. Phys. 67, 1 (2004).

${ }^{48}$ W. H. Press, S. A. Teukolsky, W. T. Vetterling, and B. P. Flannery, Numerical Recipes in C: The Art of Scientific Computing (Cambridge University Press, New York, 1992).

${ }^{49}$ NIST/SEMATECH e-Handbook of Statistical Methods, http:// www.itl.nist.gov/div898/handbook/, 6/01/2003.

${ }^{50}$ G. Schubert and H. Fehske, Lect. Notes Phys. 762, 135 (2009). 